\title{
Discrimination of loudness similarity ${ }^{1}$
}

\author{
JACOB BECK, UNIVERSITY OF OREGON \\ WILLIAM A. SHAW, UNIVERSITY OF PENNSYLVANIA
}

Interval and ratio scale values were derived from measures of variability in the discrimination of loudness similarity. The interval values are linearly related to interval values based on equisection judgments (Garner, 1954), cumulating jnds (Riesz, 1933), and the dispersion of absolute judgments (Garner, 1952). Scale values based on three diverse discriminability procedures and on the method of equisection are thus in good agreement. The ratio values, however, are at variance with ratio values determined by direct ratio estimations (Stevens, 1955).

Judgmental variability caused by the imperfect discrimination of loudness similarity may be used to generate interval (Guilford, 1954) and ratio (Luce, 1961) scales of loudness. Given three loudnesses, $L_{i}, L_{j}$, and $L_{k}, O$ is required to judge whether the intermediate loudness, $\mathrm{L}_{\mathrm{j}}$, is more similar to the low loudness, $L_{i}$, or to the high loudness, $L_{k}$. For given $L_{i}$ and $L_{k}$, the proportion of times each of several $\mathbf{L}_{j} s$ is judged similar to the reference loudnesses may be used to derive interval and ratio scale values. The present study compares the interval and ratio scale values generated from measures of variability in the discrimination of loudness similarity with loudness scales based on direct response (Garner, 1954; Stevens, 1955) and discriminability (Riesz, 1933; Garner, 1952) procedures.

\section{Method}

Each stimulus consisted of three loudnesses presented in a sequence. Each loudness lasted 1 sec and the loudnesses were separated by $1 \mathrm{sec}$. The interval between the high loudness and the low loudness was always $15 \mathrm{~dB}$. There were seven loudness ranges and five intermediate loudnesses within each range. These are shown in Table 1.

The stimuli, 1000 cps tones, were produced by a Hewlett-Packard audio oscillator and delivered via a TDH-39 earphone to the O's right ear. Before each stimulus presentation the $E$ adjusted the intensities of the three loudnesses. All timing was controlled electronically and the entire sequence was set off automatically when $\mathrm{E}$ pressed a button.

Thirteen Os served in the experiment. The Os were undergraduates at the University of Pennsylvania and were paid to participate. Each $O$ was run individually in a soundproof room. The first $10 \mathrm{Os}$ were presented with four ranges shown as Group 1 in Table 1. Five intermediate stimuli within each range made a total of 20 stimuli. The first three Os judged each stimulus triple 30 times, 15 times when the loudnesses were presented in an ascending order and 15 times when they were presented in a descending order. Observers numbered 4 through 10 judged each stimulus 34 times, 17 times in the ascending order and 17 times in the descending order. Thus the first three Os each made a total of 600 judgments, and Os 4 through 10 each made a total of 680 judgments. The total set of judgments was divided into blocks of 40. Each block of 40 judgments consisted of 20 presentations in an ascending order and 20 presentations in a descending order. The order of the presentations was randomized within each block. Each $O$ completed 120 judgments in a given sitting which lasted approximately $40 \mathrm{~min}$.

Observers numbered 10 through 13 were presented with the three additional loudness ranges shown as Group 2 in Table 1 . Thus they were presented in total with seven loudness ranges. The same procedure was followed as with os 1 through 10 . The total set of judgments, however, was now randomized in blocks of 70 consisting of the 35 stimuli presented in an ascending and a descending order. The last three Os judged each stimulus 34 times, 17 times

Table 1. Medians and Standard Deviations for Loudness Ranges in Groups 1 and 2

\begin{tabular}{clccc}
$\begin{array}{c}\text { Reference Reference } \\
\text { Loudnesses } \\
\left(L_{j} ; L_{k}\right)\end{array}$ & $\begin{array}{c}\text { Intervening Loudnesses }\left(L_{j}\right) \\
(.0002 \text { dyne/cm })\end{array}$ & $\begin{array}{c}\text { Medians in } \mathrm{dB} \\
\text { (read from ogive) }\end{array}$ & $\begin{array}{c}\text { Standard Deviations } \\
\text { in } \mathrm{dB} \text { (read } \\
\text { from ogive) }\end{array}$ \\
\hline Group 1 & $30-45 \mathrm{~dB}$ & $33.4, * 35.1,37.5,39.7,40.4^{*}$ & 36.75 & 4.50 \\
& $45-60 \mathrm{~dB}$ & $50.3, * 51.4,52.4,53.9,54.8 *$ & 52.75 & 3.50 \\
& $60-75 \mathrm{~dB}$ & $65.6,66.4,67.4,68.4,69.0 *$ & 67.75 & 2.50 \\
& $75-90 \mathrm{~dB}$ & $81.2, * 82.0,82.8,83.9,84.2$ & 84.50 & 2.50 \\
Group 2 & $38-53$ & $42.4,43.8,45.7, * 48.2,49.0$ & 45.50 & 4.0 \\
& $53-68$ & $58.7,59.6,60.8, * 61.8,62.4$ & 60.25 & 2.50 \\
& $68-83$ & $74.2,75.2, * 76.3,77.2,78.0$ & 76.50 & 2.50 \\
\hline
\end{tabular}

Note: The scale values of the loudnesses marked by an asterisk were correlated with scale values derived from cumulating jnds (Riesz, 1933) and from absolute loudness judgments (Garner, 1952). Sce text. 


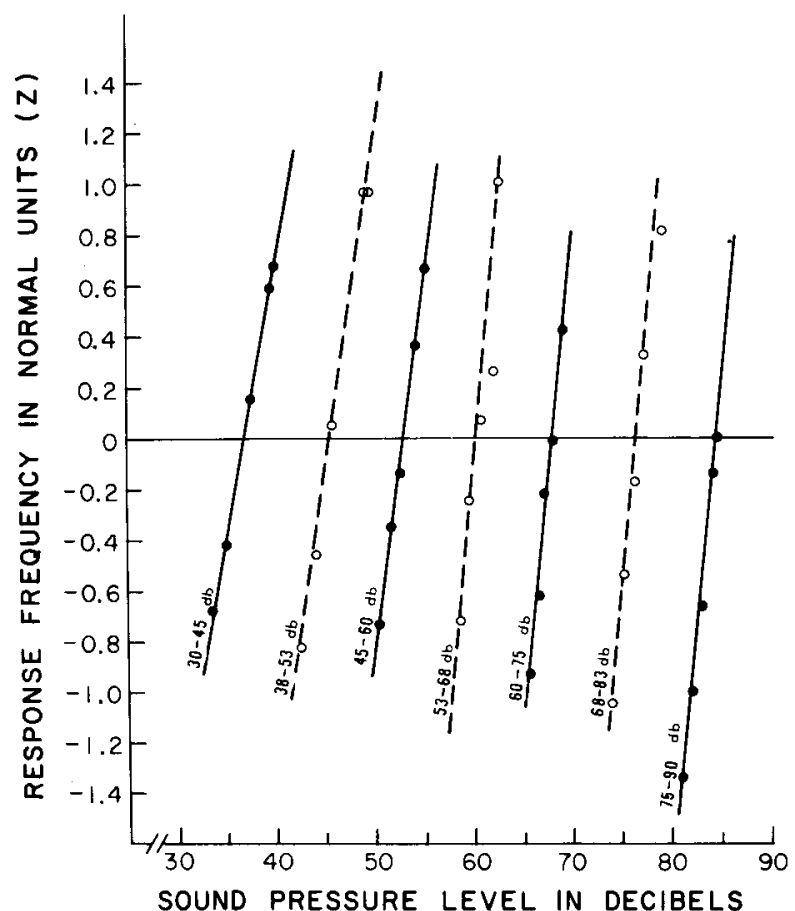

Fig. 1. The plotted points represent the frequency of times an intervening loudness, $L_{j}$, was judged more similar to the high loudness expressed in normal units $(Z)$. Each regression line was visually fitted to the data points. The low, $L_{i}$, and high, $L_{k}$, loudnesses bounding an interval are indicated next to each line. (The filled points are based on 430 judgments given by 13 os and the unfilled points on 102 judgments given by 3 os.)

in the order faint-loud and 17 times in the order loud-faint. Thus, these $O s$ each made a total of 1190 judgments.

\section{Results and Discussion}

The psychometric functions obtained are presented in Fig. 1. The abscissa is the loudness in decibels, and the ordinate is the frequency of times the intervening loudness was judged more similar to the high loudness expressed in normal units $(Z)$. The filled circles plot the $Z$ values corresponding to the mean percentages for each of the intervening loudnesses for the loudness ranges in Group 1. The open circles plot the $Z$ values for the additional loudnesses in Group 2. The fact that the data points closely conform to the visually fitted straight lines indicates that the psychometric functions are ogival.

The loudness at which the regression lines pass through the $50 \%$ point ( 0 in $\mathrm{Z}$ units) was taken as that loudness which would appear equally similar to the low and high reference loudnesses. These median loudnesses are listed in Table 1. The results indicate that the midpoints of each of the loudness intervals are close to the mean of the high and low reference loudnesses in $\mathrm{dB}$. These results are similar to those obtained when an $O$ divides a loudness interval to obtain the midpoint (Garner, 1954). A separate examination of the ascending and descending trials shows that the midpoint of an interval depends on whether the loudnesses were presented in an ascending or descending order. For example, in the 30-45 dB range, when an $O$ hears the loudnesses in an ascending order, the midpoint is $1.23 \mathrm{~dB}$ higher than when the $O$ hears the loudnesses in a descending order. Stevens (1957) has reported the same shift of the midpoint in bisection experiments, and has called the effect hysteresis. In the present study, the magnitude of hysteresis tended to vary directly with differential sensitivity. At higher intensities the relative jnd becomes smaller (Riesz, 1928). In the range 75-90 dB, the ascending midpoint is only .25 $\mathrm{dB}$ higher than the descending midpoint.

Table 1 also presents the standard deviations of the psychometric functions. The slope of the psychometric function is inversely related to the magnitude of the standard deviation. Inspection of Table 1 indicates that the standard deviation is constant for ranges in which loudness of the intervening stimuli are above $55 \mathrm{~dB}$; for ranges in which the loudness of the intervening stimuli falls below $55 \mathrm{~dB}$, the standard deviation increases. Above $55 \mathrm{~dB}$ the slopes of the psychometric functions are steeper and remain constant. The slope of the psychometric function is also inversely proportionate to the size of the jnd. Thus, this effect may be presumed to be due to the fact that the relative jnd for a $1000 \mathrm{cps}$ tone is approximately constant above $55 \mathrm{~dB}$ and increases for loudnesses below this value (Riesz, 1928).

Since the regression of the $\mathrm{Z}$ values on $\mathrm{dB}$ within each of the loudness ranges is a straight line, the $Z$ values may be taken to express the distance of each loudness from the median of the ogive function in sigma units. If we let the standard deviation become the unit of the scale (i.e., equal measures of discriminability represent equal distances on a magnitude scale of loudness), we may place all the $Z$ values on a single scale by introducing additive constants to adjust the intercept values. The intercept constants were adjusted so that two neighboring ranges were made to coincide at the midvalue between the two ranges. For example, in the $30-45 \mathrm{~dB}$ range, the highest intervening loudness was $40.4 \mathrm{~dB}$; in the 38-53 $\mathrm{dB}$ range, the lowest intervening loudness was $42.4 \mathrm{~dB}$. The scale values in these two ranges were located with respect to one another by finding the scale values for $41.4 \mathrm{~dB}$ from the regression lines shown in Fig. 1. The constant necessary to make the scale value at $41.4 \mathrm{~dB}$ in the $38-53 \mathrm{~dB}$ range equal to the scale value at $41.4 \mathrm{~dB}$ in the $30-45 \mathrm{~dB}$ range was then determined; this constant was then added to all the $Z$ values in the 38-53 dB range. The intercept values of the remaining ranges were adjusted in the same manner to yield a single psychometric function.

Table 2 shows the scale values obtained for the 


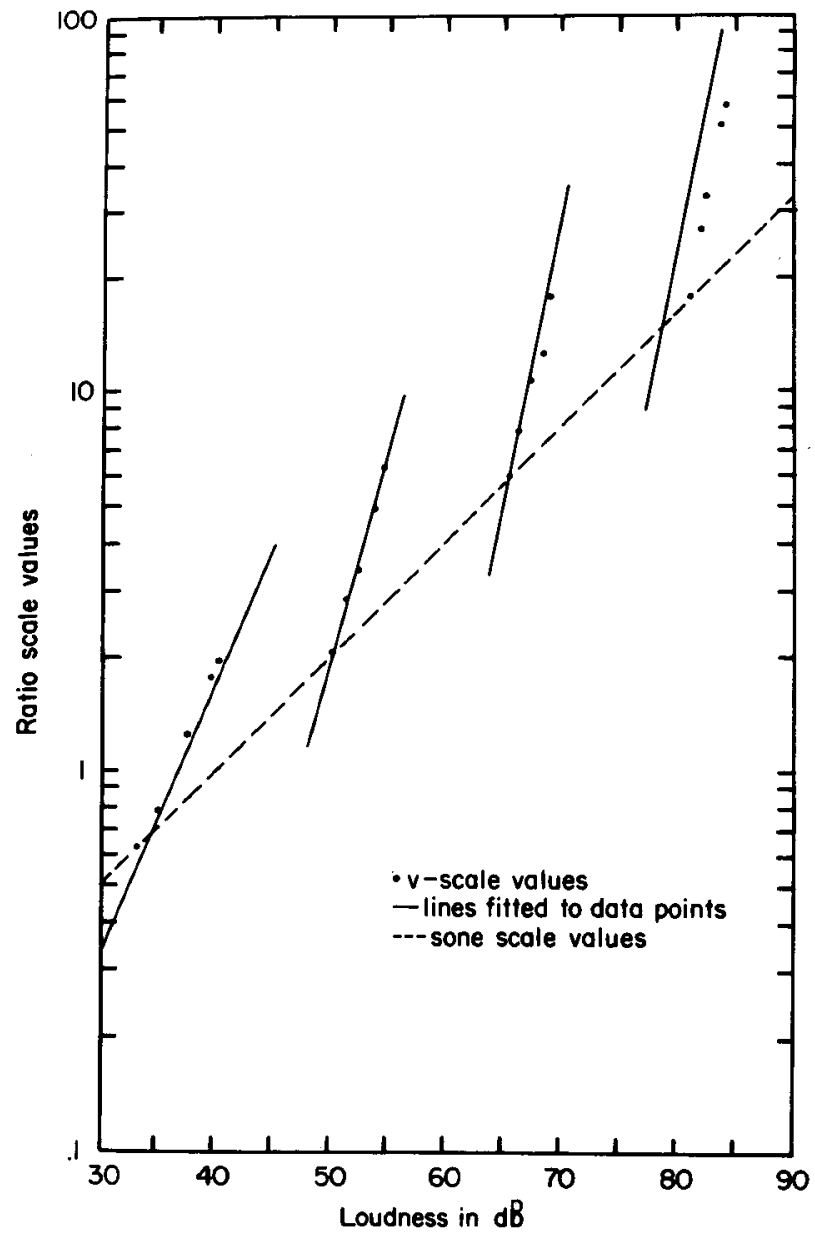

Fig. 2. The plotted points show the ratio scale values derived from the obtained proportions for the intervening loudnesses in Group 1 by the use of Luce's (1961) formula. The solid lines were visually fitted to the data points while satisfying the requirement that the product of the scale values for the reference loudnesses, $v\left(L_{i}\right)$ and $v\left(L_{k}\right)$, is equal to the constant chosen in each range (see text). description only if a scale is curved near the origin, but is approximately straight over the higher ranges. The present scale, as in the integrated jnd scale of Riesz (1933), departs from approximate linearity below $55 \mathrm{~dB}$, and the curvature cannot be rectified by the addition of a constant. It is perhaps worth noting that an additive constant will produce linearity in a log-log plot of a v-scale when discriminability varies as a linear function of intensity (Luce, 1959).

\section{References}

Garner, W. R. An equal discriminability scale for loudness judgments. J. exp. Psychol., 1952, 43, 232-238.

Gamer, W. R. A technique and scale for loudness measurements. J. Acoust. Soc. Amer., 1954, 26, 73-88.

Gamer, W. Advantages of the discriminability criterion for a loudness scale. J. Acoust. Soc. Amer., 1958, 30, 1005-1012.

Guilford, J. P. Psychometric methods. New York: McGraw-Hill, 1954.

Luce, R. D. Individual choice behavior. New York: Wiley, 1959

Luce, R. D. A choice theory analysis of similarity judgments. Psychometrika, 1961, 26, 161-163.

Luce, R. D., \& Edwards, W. The derivation of subjective scales from just noticeable differences. Psychol. Rev., 1958, 65, 222237.

Reynolds, G. S., \& Stevens, S. S. Binaural summation of loudness. J. Acoust. Soc. Amer., 1960, 32, 1337-1344.

Riesz, R. R. Differential intensity sensitivity of the ear for pure tones. Phys. Rev., 1928, 31, 867-875.

Riesz, R. R. The relation between loudness and minimum perceptible increment of intensity. J. Acoust. Soc. Amer., 1933, 4, 211216.

Stevens, S. S. The measurement of loudness, J. Acoust. Soc. Amer., 1955, 27, 815-829.

Stevens, S. S. On the psychophysical law. Psychol. Rev., 1957, $64,153-181$.

\section{Note}

1. This research was supported by ONR grant Nohr-551 (41) to the University of Pennsylvania. The preparation of this paper was supported by National Science Foundation Grant GB-5285.

(Accepted for publication October 19, 1967.)

\section{Erratum}

Bixler, E. O., Bartlett, N. R., \& Lansing, R. W. Latency of the blink reflex and stimulus intensity. Percept. \& Psychophys., 1967, 2 (11), 559-560. The statement made on line 9 and 10 under method should be corrected to read: "with an intensity loss of about $85 \% . " 1$ 